\title{
ASCA OBSERVATIONS OF CLASS I PROTOSTARS IN THE RHO OPH DARK CLOUD
}

\author{
Y. TSUBOI AND K. KOYAMA
}

Department of Physics, Graduate School of Science, Kyoto University, Sakyo-ku, Kyoto, 606-01, Japan

Y. KAMATA

Department of Astrophysics, School of Science, Nagoya University, Furo-cho, Chikusa-ku, Nagoya 464-01, Japan

AND

S. YAMAUCHI

Faculty of Humanities and Social Sciences, Iwate University, 3-18-34, Ueda, Morioka, Iwate, 020, Japan

\section{Introduction}

On 1993 August 20, we observed the Rho-Oph dark cloud and detected hard X-rays from Class I sources (Koyama et al.(1994), Kamata et al.(1997)). One of the sources (EL29) showed a flare-like variability, while another (WL6) exhibited sinusoidal variation with no large spectral change. The later would be due to a spin of the protostar. The sinusoidal period of about 1 day is shorter than spin periods of TTSs of $\sim 3-7$ day.

From these X-ray emitting YSOs, we found bipolar flows with radio observations (Sekimoto et al.(1997)). This places the sources to be protostars at the dynamical mass accretion phase. The common feature of these out-flows is that the blue and red lobes are largely overlapped, suggesting nearly pole-on geometry. On the other hand, no significant X-ray has been reported from out-flow sources, VLA 1623 (Kamata et al.(1997)), L1551 IRS5 and L1551 NE, HL Tau (Carkner et al.(1996)), all these would be edge-on systems (André et al.(1990), Ohashi et al.(1996)). Consequently, we proposed a unified picture of protostars; every protostar emits X-rays, but the X-rays can only be detected from pole-on viewing angle, where $\mathrm{X}$-rays are less absorbed by dense circumstellar disks. 
To study the time variability of the X-ray emitting Class I sources, we re-observed this region deeply.

\section{Observations \& Results}

On 1997 Mar 2-3, we observed the central region of the cloud with $100 \mathrm{ksec}$ exposure, and found that WL6 and EL29 became very faint. Instead, new hard X-ray ( $>2 \mathrm{keV}$ ) objects appeared at the positions of other Class I stars. The brightest was YLW15, on which a large flare had been detected with the ROSAT deep pointing observation (Grosso et al.(1997)). The outflow map of YLW15 (Bontemps et al.(1996)) has also a signature of pole-on configuration, confirming our unified picture.

In the hard band $(>2 \mathrm{keV})$, we detected three flares on the YLW15 with about 20 hours interval. The time interval is comparable to the spin period of the protostar WL6, which we suggested from the sinusoidal X-ray light curve. It is also comparable to the period of the inner-most Keplarian orbit $\left(r=\right.$ several $\left.\times 10^{-2} \mathrm{AU}\right)$. Accordingly, the quasi-periodicity of the flares would be related to the spin of the central star or/and rotation of the circumstellar disk.

\section{References}

André, P., Martin-Pintado, J., Montmerle, T.: 1990, $A \& A, 236,180$.

Bontemps, S., André, P., Terebey, S., Cabrit, S.: 1996, A\&\&A 311858.

Carkner, L., Feigelson, E.D., Koyama, K., et al.: 1996, ApJ, 464, 286.

Grosso, N., Montmerle, T., Feigelson, E. D., et al.: 1997, Nature, 387, 56.

Kamata, Y., Koyama, K., Tsuboi, Y., Yamauchi, S.: 1997, PASJ, 49, 461.

Koyama, K., Maeda, Y., Ozaki, M., et al.: 1994, PASJ, 46, L125.

Ohashi, N., Hayashi, M., Ho, P. T. P., et al.: 1996, ApJ, 466, 957.

Sekimoto, Y., Tatematsu, K., Umemoto, T., et al.: $1997, A \boxminus A L$, in press.

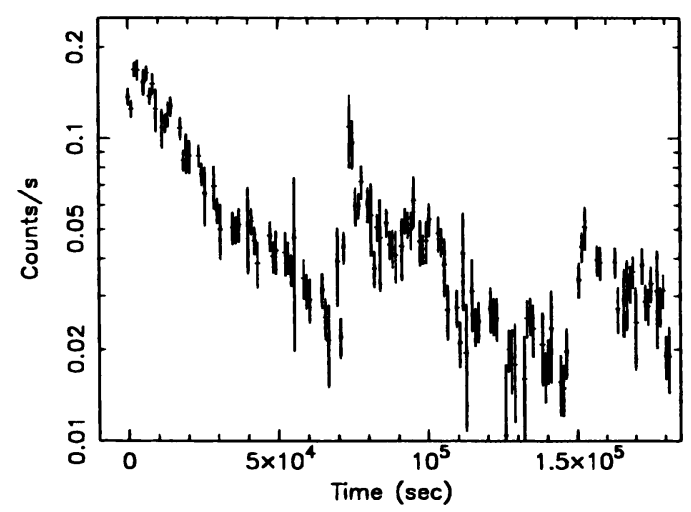

Figure 1. Lightcurve of YLW15 in $2-10 \mathrm{keV}$ band (GIS $2+3$ ). Each time bin width is $1024 \mathrm{sec}$. 